\title{
Reactive Arthritis Following Intravesical Instillation of Bacillus Calmette-Guerin
}

\author{
Selin HASSAN, Peyman YALÇIN, Şebnem ATAMAN, Nurben SÜLDÜR \\ Department of Physical Medicine and Rehabilitation, Medical Faculty of Ankara University, Ankara, Turkey
}

\begin{abstract}
Reactive arthritis is a seronegative, HLA-B27-linked spondyloarthropathy which occurs as a result of a pathogen, often of gastrointestinal or genitourinary origin, and triggers a cell-mediated immune response. In this article, we present a 64-yearold male case who developed reactive arthritis with fever and asymmetric oligoarthritis following treatment with intravesical Bacillus Calmette-Guerin (BCG) for bladder carcinoma.

Keywords: Bacillus Calmette-Guerin; HLA-B27 antigen; reactive arthritis.
\end{abstract}

Reactive arthritis is a seronegative, human leukocyte antigen (HLA)-B27-linked spondyloarthropathy ${ }^{1}$ in which the onset of acute joint pain and inflammation takes place following the provocation of autoimmune responses. ${ }^{2}$ The infectious pathogen, often of gastrointestinal or genitourinary origin, ${ }^{3}$ induces a cell-mediated immunity. In addition, it is also believed that antigen-specific CD4 and CD8 T cells are activated within the articular cavity, triggering an inflammatory response within the tissues. ${ }^{4}$ In many cases, by the time the patient presents with symptoms, the trigger has either been cured or has gone into remission, making it difficult to determine the original etiology.

\section{CASE REPORT}

A 64-year-old male initially presented with a sevenweek history of fever and lower back pain. This was followed by swelling of the right knee and left ankle along with complaints of intermittent wrist pain and stiffness; however the patient denied having any gastrointestinal or genitourinary symptoms. Additionally, his past medical history included a diagnosis of low-grade bladder carcinoma in 1998 for which he received six doses of intravesical Bacillus Calmette-Guerin (BCG). Afterwards, the patient had a follow-up cystoscopy every six months, and intravesical mitomycin $C$ was used to treat each recurrence of a low-grade tumor. In 2011, following another tumor recurrence, the patient was treated with weekly doses of intravesical BCG, the fourth dose of which he received two weeks before the onset of the aforementioned symptoms.

The patient was admitted to the hospital with a preliminary diagnosis of reactive arthritis and was treated with sulfasalazine $500 \mathrm{mg}$ once daily and a non-steroidal anti-inflammatory drug (NSAID). He was also started on antibiotic therapy (a quinolone derivative), and an intra-articular betamethasone injection was administered to his right knee. The patient's pain and swelling responded well to the treatment, and he was discharged two days later.

Received: September 20, 2012 Accepted: February 21, 2013

Correspondence: Selin Hassan, M.D. Ankara Üniversitesi Tıp Fakültesi, Fiziksel Tıp ve Rehabilitasyon Anabilim Dalı, 06100 Sıhhiye, Ankara, Turkey. Tel: +90 530 - 9786262 e-mail: selinhassan@hotmail.com 
The patient returned to the hospital after 10 days complaining of the same symptoms. An ultrasound of the right knee revealed areas of inflammation, and synovial fluid was aspirated and sent for sampling. However, the culture and microscopy were normal. Following this, the patient was given another injection of intraarticular betamethasone into right knee, and the patient's sulfasalazine dosage was increased to $1 \mathrm{~g}$ twice daily. Again he responded well to the treatment and was subsequently discharged.

One month later, the patient returned with the same complaints. An examination of the musculoskeletal system revealed normal joint range of motion ( $\mathrm{ROM})$, including the lower back. A minimal increase in temperature was felt when touching the skin, and we noted patellar shock positivity of the right knee joint. In addition, left ankle ROM was normal with minimal swelling at the lateral border, and sacroiliac compression and Mennel tests were negative.

The patient was then admitted for further investigation. From an analytical point of view, there was an increase in acute phase reactants [C-reactive protein (CRP) $95.2 \mathrm{mg} / \mathrm{L}$ and erythrocyte sedimentation rate (ESR) $87 \mathrm{~mm} / \mathrm{hr}$. Microcytic anemia was also present (hemoglobin $11.2 \mathrm{~g} / \mathrm{dL}$, mean erythrocyte volume $79.4 \mathrm{fl}$,

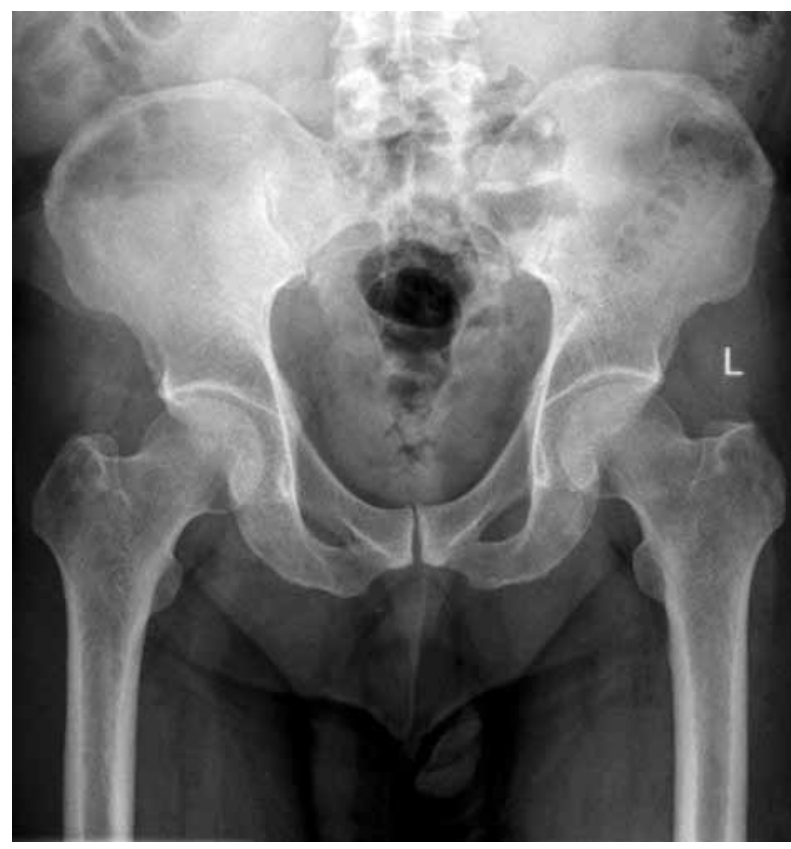

Figure 1. Pelvis X-ray showing degenerative changes. and iron $37 \mu \mathrm{g} / \mathrm{dl}$ ), but the ferritin and serum levels were normal as were the total iron binding capacity and transferrin saturation rate. Serum protein electrophoresis revealed a decrease in albumin (47.3\%), whereas the alpha 1 (16.1\%), alpha 2 (14.9\%), and gamma globulin (21.3\%) levels were higher. Other immunological tests determined that the rheumatoid factor (RF), anticyclic citrullinated peptide (anti-CCP), antinuclear antibody (ANA), complement, and anti-doublestranded deoxyribonucleic acid (anti-dsDNA) levels were normal, and the serum uric acid levels were also within normal limits. Furthermore, the primary and secondary tuberculosis screening tests were negative, and the serology tests for Brucella, Chlamydia, the human immunodeficiency virus (HIV), and hepatitis $B$ and $C$ were negative as well. Human leukocyte antigen typing revealed the presence of $\mathrm{A} 1, \mathrm{~A} 2, \mathrm{~B} 41, \mathrm{~B} 57, \mathrm{Cw} 6 / 7$, DR11, and DR13 while a urinary system ultrasound and an intravenous pyelogram were normal. An $\mathrm{X}$-ray of the pelvis showed degenerative changes (Figure 1), and because of the patient's ongoing lower back pain and peripheral joint swelling, magnetic resonance imaging (MRI) of the sacroiliac joints was performed, which showed periarticular edema of the sacrum in the anterior segment along with a slight increase in sclerosis of the right sacroiliac joint in the anterior superior segment.

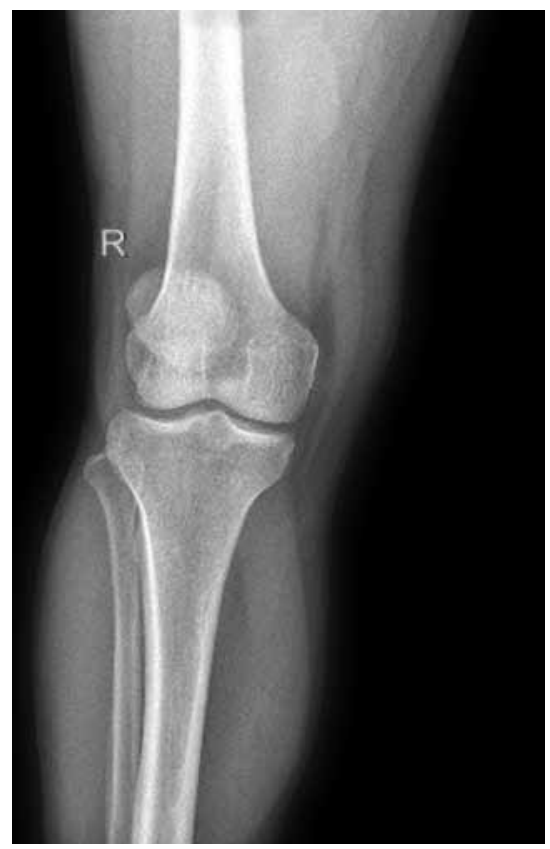

Figure 2. Right knee joint X-ray. 


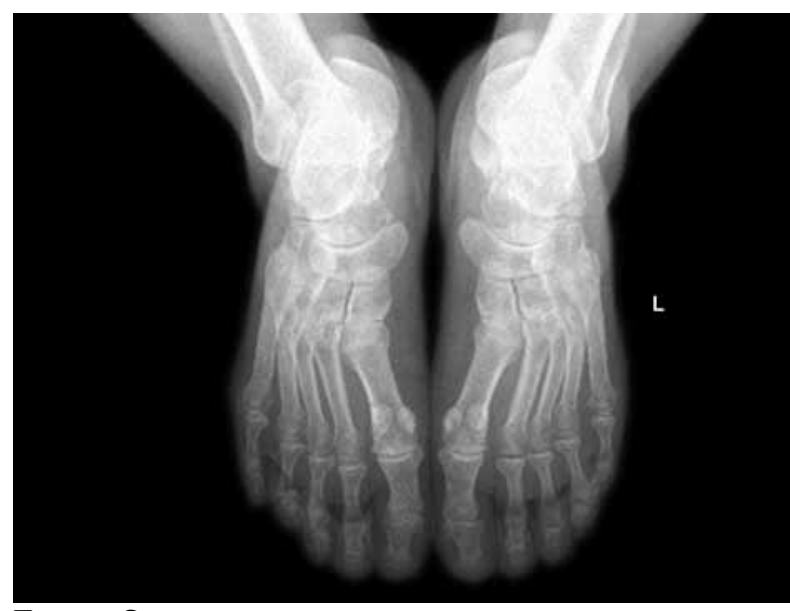

Figure 3. Feet X-ray showing osteodegenerative change.

Slight edema of the left periarticular sacral area was also seen in the posterior segment. We then recommended that the MRI findings be interpreted in accordance with the clinical picture.

The bilateral knee radiographs of the patient were normal (Figure 2), but those of the feet revealed osteodegenerative changes (Figure 3) and bilateral heel spurs (Figure 4).

The onset of asymmetrical oligoarthritis following the patient's treatment with BCG and the exclusion of other etiologies led to a confirmed diagnosis of reactive arthritis. Throughout his admission, the patient continued to receive treatment with sulfasalazine with no positive results. Therefore, once the diagnosis was confirmed, the patient was started on methotrexate $15 \mathrm{mg}$ /week, but no oral corticosteroid was given because of his earlier treatment with live attenuated BCG. The patient was discharged and outpatient follow-up appointments were scheduled. He continued treatment with methotrexate for a total of two months but then voluntarily stopped taking all of his medications because of symptom regression. At his two-month, post-discharge follow-up, the patient no longer complained of back and joint pain, and there was no swelling. An examination also revealed no signs of arthritis. His hemoglobin level had increased to $12.8 \mathrm{~g} / \mathrm{dl}$, and his CRP levels $(17.9 \mathrm{mg} / \mathrm{L})$ and ESR $(35 \mathrm{~mm} / \mathrm{hr})$ had fallen. At eight months post-discharge, the patient was no longer anemic ( $\mathrm{Hb} 15.1 \mathrm{~g} / \mathrm{dl})$, and the CRP levels $(2.89 \mathrm{mg} / \mathrm{L})$ and ESR $(3 \mathrm{~mm} / \mathrm{hr})$ had normalized.

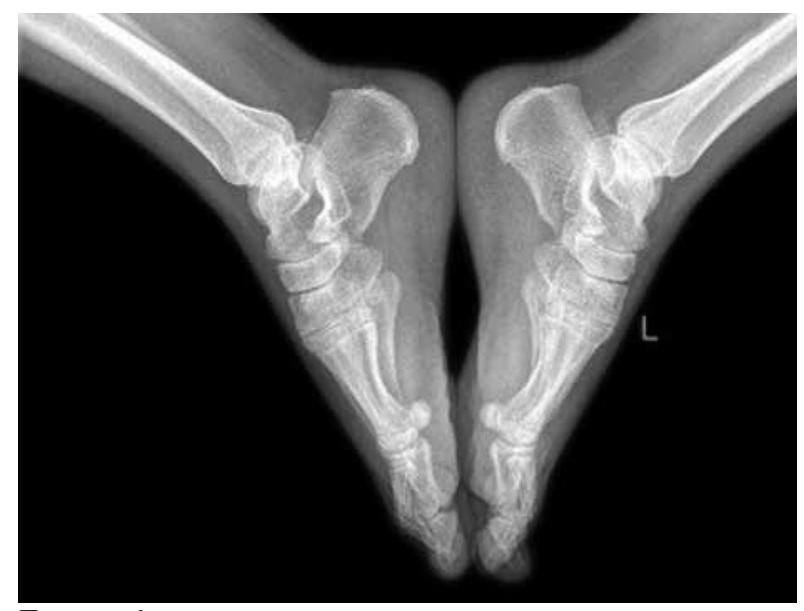

Figure 4. Feet X-ray showing bilateral heel spurs.

\section{DISCUSSION}

Intravesical instillation of BCG has been used in the treatment of intermediate and high-grade bladder carcinoma in situ since 1976 and has been proven to be safe and effective. ${ }^{5}$ Its local anti-tumor effects suggest that its immunomodulatory activity occurs mainly at the site of instillation. Although the musculoskeletal side effects of this type of treatment are well known, they have rarely been reported. ${ }^{6}$

Findings suggestive of reactive arthritis, as opposed to septic arthritis, include the onset of oligoarticular or polyarticular arthritis a few weeks after the last BCG instillation. Even so, joint fluid analysis is imperative in order to rule out septic arthritis. ${ }^{7}$ In the majority of cases, knee, ankle, and wrist involvement has also been reported anywhere from a few days to five months after the first instillation, and further instillations aggravate the arthritis. This is also accompanied by fever and/or HLA-B27 positivity in over half of cases. Moreover, spinal pain has been reported in 19\% of the patients with reactive arthritis and 14\% have had sacroiliac involvement. $^{\text {? }}$

Tinazzi et al. ${ }^{8}$ conducted a systematic review of patients with arthralgia and arthritis following BCG immunotherapy and identified 61 cases of musculoskeletal complications related to intravesical BCG instillation. These included pain and arthritis in 64\%, Reiter's syndrome in 24\%, arthritis and fever in $4 \%$, peripheral arthritis in patients with ankylosing spondylitis (AS) in 4\%, psoriatic arthritis 
(PsA) in 2\%, and Sjögrens syndrome in 2\%. In some cases, remission was achieved by discontinuing the BCG treatment and applying no other treatment or by administering NSAIDs alone. In contrast, others needed corticosteroid or immunosuppressive treatment.

In patients with reactive arthritis, laboratory investigations usually show non-specific signs of inflammation, joint fluid analyses generally reveal a predominance of polymorphonuclear cells, and radiographs reveal no abnormal findings. ${ }^{9}$ The pathophysiology by which the instillation of BCG results in reactive arthritis is unknown. One possibility that has been suggested is molecular mimicry. This occurs when HLA-B27 presents arthritogenic microbial peptides to T-cells, thereby stimulating an auto-immune response. ${ }^{10}$

Corticosteroids and NSAIDs have widely been used in the treatment of reactive arthritis, and while this treatment has been clinically effective, there is little clinical data to support this. Interestingly, in this case, treatment with NSAIDs, intra-articular corticosteroids, and sulfasalazine was ineffective, whereas methotrexate was efficacious. Even though treatment with methotrexate has previously been advocated, for patients with reactive arthritis, we believe that further evaluation is needed. ${ }^{10}$

\section{Conclusion}

Although rare, reactive arthritis secondary to treatment with intravesical BCG should be considered in patients presenting with arthralgia and arthritis. In addition, the presence of HLA-B27 positivity has been noted in over half of these cases, and HLA DR1, DR3, and DR4 may also be present. In general, patients with reactive arthritis should be treated with drug therapy, which will likely yield favorable outcomes.

\section{Declaration of conflicting interests}

The authors declared no conflicts of interest with respect to the authorship and/or publication of this article.

\section{Funding}

The authors received no financial support for the research and/or authorship of this article.

\section{REFERENCES}

1. Repo ML, Koivuniemi R, Breban M. Infections and arthritis. In. Bijlsma JWJ, editor. EULAR textbook on rheumatic diseases. 1st ed. London: BMJ Group; 2012. p. 335-6.

2 Carter JD. Reactive arthritis: defined etiologies, emerging pathophysiology, and unresolved treatment. Infect Dis Clin North Am 2006;20:827-47.

3. Harms RW, Berge KG, Hagen PT, Litin SC, Sheps SG. Reactive arthritis. Mayo Clinic [serial online] 2011. Accessed 06/06/2012. Available from: http://www. mayoclinic.com/health/reactive-arthritis/DS00486

4. Kim JY, Lee JH, Yoon IeN. Macular infarction associated with reactive arthritis. Korean J Ophthalmol 2010;24:310-3.

5. Alexandroff AB, Jackson AM, O'Donnell MA, James K. BCG immunotherapy of bladder cancer: 20 years on. Lancet 1999;353:1689-94.

6. Librenjak D, Novakovi ZS, Milosti K. Carcinoma in situ of urinary bladder: incidence, treatment and clinical outcomes during ten-year follow-up. Acta Clin Croat 2012;51:201-7.

7. Clavel G, Grados F, Lefauveau P, Fardellone P. Osteoarticular side effects of BCG therapy. Joint Bone Spine 2006;73:24-8.

8. Tinazzi E, Ficarra V, Simeoni S, Artibani W, Lunardi C. Reactive arthritis following BCG immunotherapy for urinary bladder carcinoma: a systematic review. Rheumatol Int 2006;26:481-8.

9. Pardalidis NP, Papatsoris AG, Kosmaoglou EV, Georganas $C$. Two cases of acute polyarthritis secondary to intravesical BCG adjuvant therapy for superficial bladder cancer. Clin Rheumatol 2002;21:536-7.

10. Carter JD. Treating reactive arthritis: insights for the clinician. Ther Adv Musculoskelet Dis 2010;2:45-54. 